\title{
Laparoscopic approach in the treatment of large epiphrenic esophageal diverticulum
}

\author{
Virgilijus Beiša, Mindaugas Kvietkauskas, Augustas Beiša, Kęstutis Strupas \\ Center of Abdominal Surgery, Vilnius University, Vilnius, Lithuania
}

Videosurgery Miniinv 2015; 10 (4): 584-588

DOI: $10.5114 /$ wiitm.2015.56407

\begin{abstract}
Epiphrenic diverticulum of the lower third of the esophagus is a relatively rare disorder. We present the case of a large, $7.5 \mathrm{~cm}$ diameter esophageal epiphrenic diverticulum treated by the laparoscopic approach. Surgery was indicated by the severity of the patient's symptoms and size of the diverticulum. A laparoscopic transhiatal diverticulectomy with a myotomy and Dor fundoplication was carried out. The overall operative time was $180 \mathrm{~min}$. The patient tolerated the surgery well and was discharged from hospital 4 days after the surgery. From the $10^{\text {th }}$ postoperative day the patient resumed a regular diet. Four weeks after the operation the patient had no complaints, symptoms of dysphagia or vomiting. The laparoscopic approach in the treatment of a large, $7.5 \mathrm{~cm}$ epiphrenic diverticulum of the esophagus is feasible, safe and well tolerated by the patient.
\end{abstract}

Key words: epiphrenic diverticulum, esophageal diverticulum, laparoscopic esophageal surgery, transhiatal approach.

\section{Introduction}

Epiphrenic diverticulum is a relatively rare disorder of the lower third of the esophagus, which is defined as mucosa and submucosa herniation through the muscular layers of the esophageal wall [1-3]. Usually it is located at the right side of the esophagus $[4,5]$. There is an estimated prevalence of $0.015 \%$ in the United States, $0.77 \%$ in Japan and up to $2 \%$ in Europe, but the true incidence is unknown as only $15-20 \%$ of patients are symptomatic, while a great number of cases are diagnosed incidentally during radiographic or endoscopic examinations performed for other reasons $[6,7]$. For the pathophysiology of esophageal diverticulum, muscle weakness of the esophagus, incomplete relaxation of the esophageal sphincter and increased pharyngeal pressure are important $[2,8]$. The primary symptoms are dysphagia, regurgitation, heartburn, chest pain, nausea and vomiting in the majority of patients $[5,9]$. There are a few minimally invasive approach options: laparoscopy or thoracoscopy [8-12]. Standard treatment of the epiphrenic diverticulum includes diverticulectomy, esophageal myotomy and fundoplication [9, 10]. Still there is no consensus on the type of surgical access, the length of the myotomy, or the type of antireflux technique [6].

We report the case of a laparoscopic transhiatal approach to a large esophageal epiphrenic diverticulum.

\section{Case report}

A 68-year-old woman was admitted to our hospital. She complained of epigastric pain that occurred after eating, bloating, nausea and vomiting. The preoperative study included barium swallow and esophagoscopy. The initial diagnosis was performed by barium esophagram, which revealed an epiphrenic diverticulum located on the right side and in the low- 


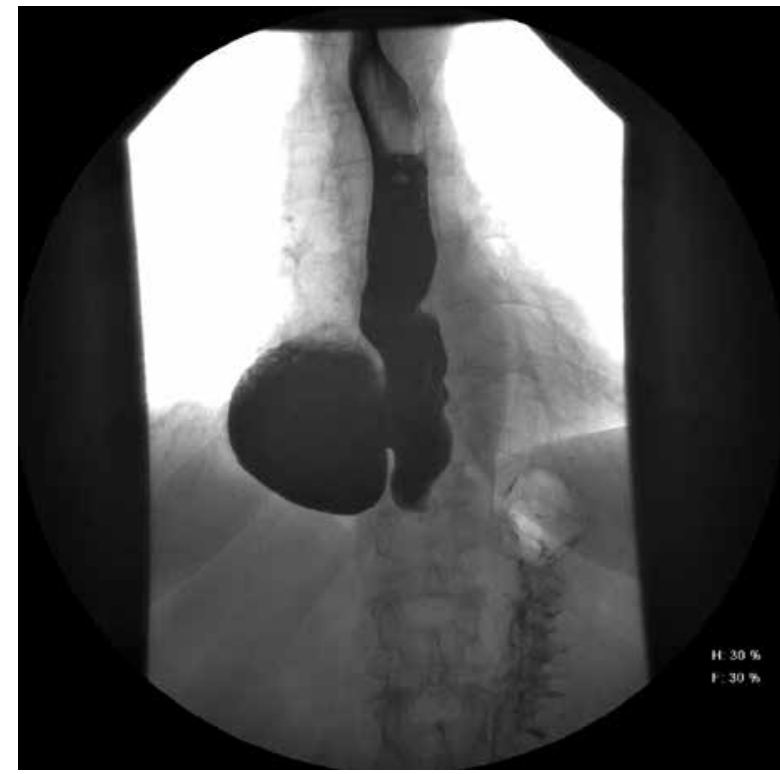

Photo 1. Preoperative barium swallow examination

er third of the esophagus (Photo 1). The diverticulum measured $7.5 \mathrm{~cm}$ in length and $6.2 \mathrm{~cm}$ in width; the neck was $2 \mathrm{~cm}$ in length. Contrast medium was retained in the diverticulum all the time during examination. The contrast medium emptied slowly from the diverticulum through the esophagus to the stomach. Also esophageal stasis above the diverticulum was found. Esophagogastroscopy was performed to exclude any diverticular ulceration or neoplastic strictures. A large epiphrenic diverticulum $33-34 \mathrm{~cm}$ from incisors with a mouth of $4 \mathrm{~cm}$ and a few erosions in the antrum of the stomach were established. In order to exclude malignancy of the esophagus the patient was referred for a neck and chest spiral computed tomography (CT) scan. It confirmed the diagnosis, showing a $7.5 \mathrm{~cm} \times 6 \mathrm{~cm}$ diverticulum with a $4 \mathrm{~cm}$ mouth and revealing no evidence of other masses or sign of malignancy (Photo 2).

Surgery was indicated by the severity of the patient's symptoms and size of the diverticulum. A laparoscopic transhiatal diverticulectomy, myotomy and Dor fundoplication were planned.

Surgery was performed under general anesthesia with orotracheal intubation, by the laparoscopic approach. The patient was placed in a reverse Trendelenburg (30-degree) supine position with legs spread. The surgeon stood between the patient's legs. A Veress needle was inserted $5 \mathrm{~cm}$ above the umbilicus. To form the pneumoperitoneum, $\mathrm{CO}_{2}$ at $10 \mathrm{~mm} \mathrm{Hg}$ was used. The trocar and video laparo-

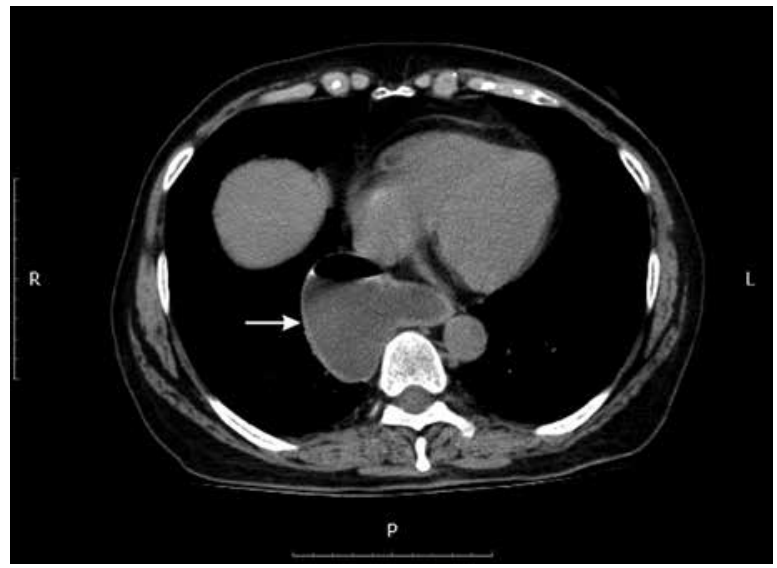

Photo 2. Preoperative spiral computed tomographic (CT) scan

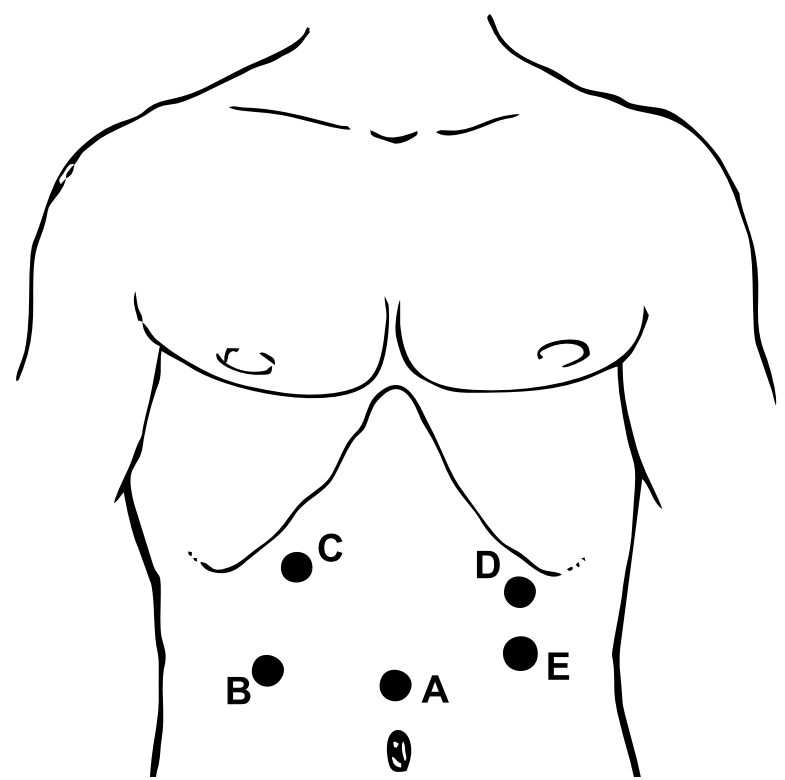

Figure 1. Positioning of the trocars. A $-10 \mathrm{~mm}$, $\mathrm{B}-10 \mathrm{~mm}, \mathrm{C}-10 \mathrm{~mm}, \mathrm{D}-10 \mathrm{~mm}, \mathrm{E}-12 \mathrm{~mm}$

scope were placed. Under the control of the laparoscope, two trocars (10 $\mathrm{mm}$ and $12 \mathrm{~mm}$ ) on the left side of the abdomen and two $10 \mathrm{~mm}$ trocars on the right side of the abdomen were inserted (Figure 1). The first assistant was at the left side of the patient and was holding the video laparoscope and surgical instrument, which was placed in the left $10 \mathrm{~mm}$ trocar. The second assistant was at the right side of the patient and was lifting up and holding the left lobe of the liver. After the esophagogastric junction was indentified, the operation started with mobilization of the abdominal esophagus from the diaphragmatic pillars and dissection of the diaphragmatic cruses. 


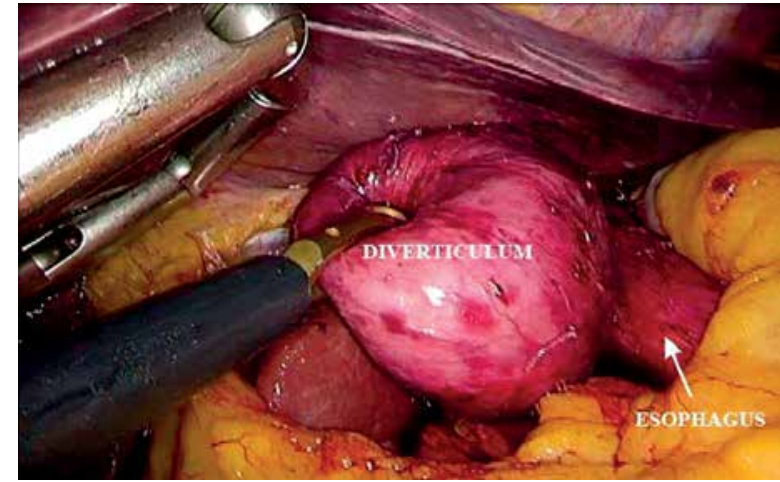

Photo 3. Dissected diverticulum

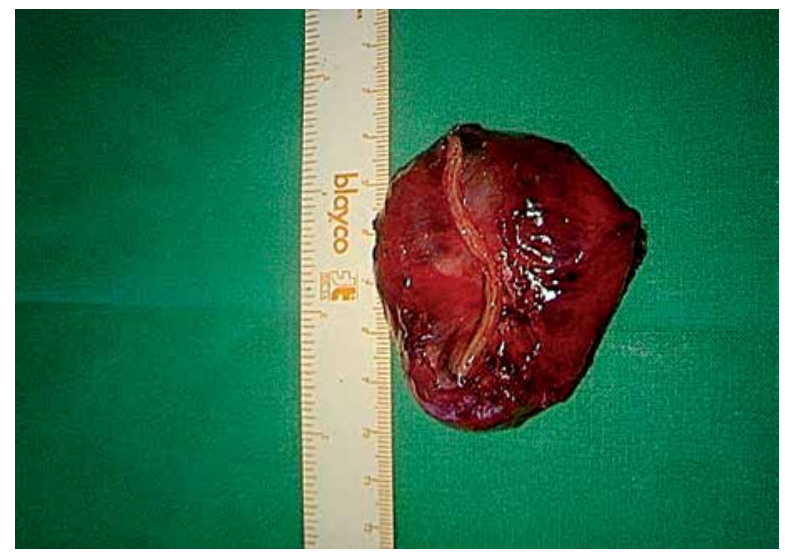

Photo 5. Resected diverticulum

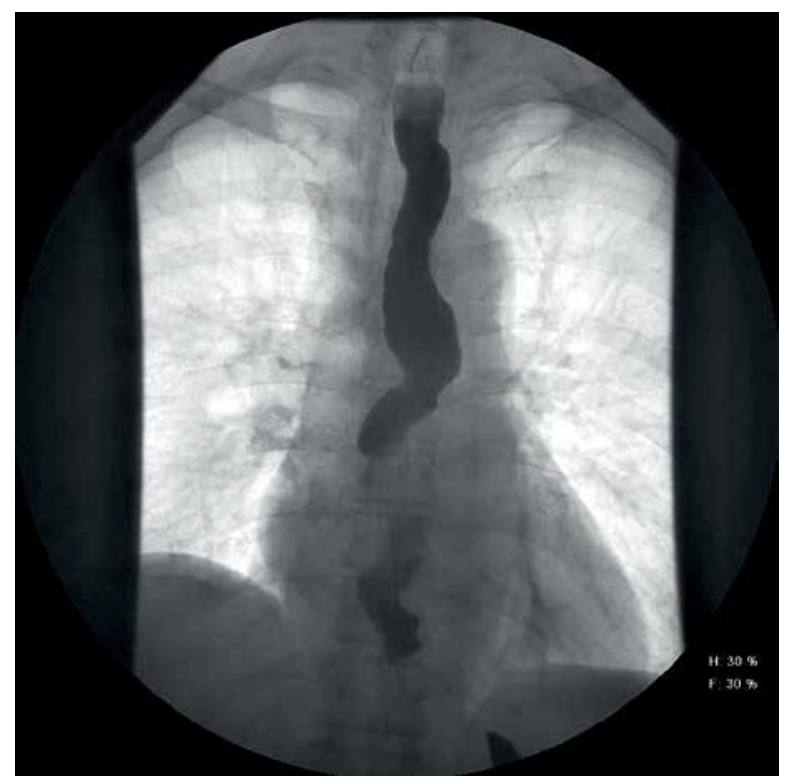

Photo 6. Postoperative esophagogram with Gastrografin swallow

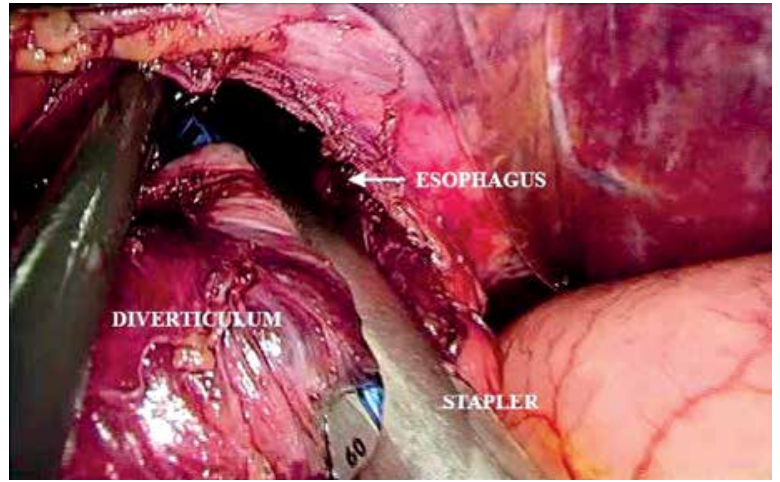

Photo 4. Endoscopic stapler placed across the neck of the diverticulum

Harmonic ACE curved shears were used. Once a wide retroesophageal space was created, gentle traction was applied to the esophagus using a retractor. The gastroesophageal junction was then encircled with umbilical tape to aid with traction during the mediastinal dissection. Then, epiphrenic diverticulum of the esophagus was mobilized from the mediastinum about $7 \mathrm{~cm}$ above the diaphragmatic cruses (Photo 3). After preparation of the diverticular pouch was completed, the $4 \mathrm{~cm}$ long diverticulum neck was sewn with the endoscopic linear surgical stapler Echelon FLEX 60 under intraoperative esophagogastroscopic control (Photo 4). The resected diverticulum was inserted in a retrieval bag. No evidence of esophageal stricture was observed, and the operation continued with esophageal myotomy $5 \mathrm{~cm}$ above and $3 \mathrm{~cm}$ below the gastroesophageal junction. After myotomy was performed, a test with gas showed no leaks from the staple line. The procedure was completed with Dor fundoplication. Ethibond 3/0 interrupted stitches were used. The bottom of the anterior stomach wall was sewn to the myotomy edges with Ethibond 2/0 interrupted stitches from both sides. To close the diaphragmatic lesion the upper stitches were sewn to the diaphragmatic cruses. The retrieval bag with the diverticulum was removed through the $12 \mathrm{~mm}$ port previously placed for the stapler. The abdominal cavity was not drained, and the trocar wounds were sutured. The resected pouch was sent to the laboratory for histological analysis (Photo 5).

The patient tolerated the surgery well. The overall operative time was $180 \mathrm{~min}$. On the first postoperative day an esophagogram with Gastrografin swallow (Photo 6) and chest X-ray was performed. It excluded any defects of suture line integrity and pneumothorax. The patient had no complaints of dysphagia or 
vomiting. A liquid diet was prescribed for 3 days; it was tolerated without any pain, regurgitation or dysphagia. On the $4^{\text {th }}$ day after surgery the patient was discharged from hospital in a good state of health with recommendations for a soft diet for 5 days.

The results of histological analysis showed a $7.5 \mathrm{~cm} \times 6 \mathrm{~cm}$ esophageal diverticulum with $0.3 \mathrm{~cm}$ thickness of the wall. It was covered with stratified squamous cell epithelium, had some focal erosions, and was infiltrated with monomorphic leukocytes. Partial or total atrophy of the muscular layer of the diverticulum was observed.

From the $10^{\text {th }}$ postoperative day the patient resumed a regular diet. Four weeks after the operation the patient had no complaints, symptoms of dysphagia or vomiting.

\section{Discussion}

Most esophageal diverticula are acquired lesions that present in 60-70-year-old male patients. Epiphrenic esophageal diverticula are located up to $10 \mathrm{~cm}$ from the gastroesophageal junction and account for about $15 \%$ of all esophageal diverticula [4].

The first successful diverticulectomy was carried out by von Bergman in 1892 though surgical techniques had been postulated for decades [13]. In 1998 Rosati et al. first reported the results of a diverticulectomy, myotomy, and Dor fundoplication through a laparoscopic approach in four patients with epiphrenic diverticulum [10]. Diagnostics of epiphrenic diverticula based on symptoms caused by the underlying motility disorder can be difficult [9]. Barium swallow is probably the most important test to diagnose the esophageal diverticulum. This test determines the diverticulum size, neck measurements, the location and the distance from the gastroesophageal junction [2, 8, 9, 12, 14, 15]. Upper digestive endoscopy is mandatory in all cases when a diverticulum is suspected in order to diagnose the diverticulum and rule out malignancy and associated diseases. Other tests such as manometry and $\mathrm{pH}$ monitoring are indicated in symptomatic patients and show esophageal motor disorders, but not the main causes [8, 12]. They can initiate the need of endoscopy and barium swallow tests to establish the esophageal diverticulum. Chest CT scan is an uncommon test for this disorder, but very valuable for revealing evidence of esophageal malignancy $[11,16]$.
The indications for the treatment of epiphrenic esophageal diverticulum are not unanimous. While some authors believe that only symptomatic and large diverticula ( $>4 \mathrm{~cm}$ ) must be treated, other authors advise operative intervention even in the $a b$ sence of symptoms, to protect patients from the risk of aspiration [5, 12, 17].

Nowadays the three most used approach options are laparoscopy, thoracoscopy and thoracotomy. The transthoracic approach should be used for patients who have giant or very high diverticula, or when the technical expertise with minimally invasive techniques is not available [12]. However, this method is associated with up to $20 \%$ increased staple-line leakage and postoperative morbidity compared with the laparoscopic approach [3, 7]. The laparoscopic approach to epiphrenic diverticula should be considered today as the surgical treatment of choice for most patients [12, 18, 19]. Technical factors support this choice, including better visualization of the esophagogastric junction, easier myotomy, performance of antireflux wrap and better alignment of the stapler cartridge to the longitudinal axis of the esophagus. Furthermore, the laparoscopic approach reduces postoperative pain. Potential disadvantages of this method could be a difficult dissection of the upper part of the diverticular neck and the major risks of pleural lesions [1, 9].

Whatever the surgical approach, three main principles must be respected: diverticulectomy, long esophagogastric myotomy and a partial non-obstructive anti-reflux procedure [2]. Diverticulectomy alone has been associated with increased rates of recurrences and suture line leaks [9]. A recent study by Rice et al. demonstrated histologic abnormalities of the esophageal myenteric plexus in almost $80 \%$ of these patients, although this was not strictly related to a specific motility disorder [7, 20]. Nowadays there is general agreement that it is necessary to perform a myotomy in association with diverticulectomy to correct the underlying motor dysfunction. Some authors recommend routine fundoplication in every patient with myotomy for esophageal diverticulum [1]. However, controversy exists with regard to the choice of operation between Nissen, Toupet or Dor fundoplication and the length of the myotomy. We preferred the Dor fundoplication, because it is easy to perform, prevents reflux, secures suture line and does not increase the intraesophageal pressure. We performed myotomy $5 \mathrm{~cm}$ above and $3 \mathrm{~cm}$ be- 
low the gastroesophageal junction. We have found intraoperative endoscopy to be very useful, and we believe that it should be performed in every case. It can be used for aspiration of residual food, correct identification of the diverticulum borders, dissection and resection of the diverticulum, checking for staple-line leakage and the myotomy [1].

To sum up, there is a lack of randomized studies that compare minimally invasive approach options for diverticulectomy. In laparoscopic treatment of esophageal diverticulum, reviews present only up to twenty operation cases. There is no common agreement on patient selection and choice of surgical technique. Even though the laparoscopic approach to epiphrenic diverticula is challenging for the surgeon, technical factors, the lower rate of complications, reduced postoperative pain and early discharge from hospital are factors which support the choice of this method.

\section{Conclusions}

A large, $7.5 \mathrm{~cm}$ epiphrenic diverticulum of the esophagus can be successfully removed through the laparoscopic approach. We have found this method to be safe and well tolerated by the patient.

\section{Conflict of interest}

The authors declare no conflicts of interest.

\section{References}

1. Hirano Y, Takeuchi H, Oyama T, et al. Minimally invasive surgery for esophageal epiphrenic diverticulum: the results of 133 patients in 25 published series and our experience. Surg Today 2013; 43: 1-7.

2. Gust L, De Lesquen H, Ouattara M, et al. Open surgical management of oesophageal diverticulum. Multimed Man Cardiothorac Surg 2015; 2015. pii: mmv011. doi: 10.1093/mmcts/mmv011.

3. Onwugbufor MT, Obirieze AC, Ortega G, et al. Surgical management of esophageal diverticulum: a review of the Nationwide Inpatient Sample database. J Surg Res 2013; 184: 120-5.

4. Tse CS, Parikh ND. An uncommon source of upper gastrointestinal bleeding: epiphrenic esophageal diverticulum. Gastroenterol Rep 2015. pii: gov043. [Epub ahead of print].

5. Nehra D, Lord RV, DeMeester TR, et al. Physiologic basis for the treatment of epiphrenic diverticulum. Ann Surg 2002; 235: 346-54.

6. Gonzalez-Calatayud M, Targarona EM, Balague C, et al. Minimally invasive therapy for epiphrenic diverticula: systematic review of literature and report of six cases. J Minim Access Surg 2014; 10: 169-74.

7. Rossetti G, Fei L, del Genio G, et al. Epiphrenic diverticula mini-invasive surgery: a challenge for expert surgeons-person- al experience and review of the literature. Scan J Surg 2013; 102: $129-35$

8. Herbella FM, Patti MG. Modern pathophysiology and treatment of esophageal diverticula. Langenbecks Arch Surg 2012; 397: 29-35.

9. Silecchia G, Casella G, Recchia CL, et al. Laparoscopic transhiatal treatment of large epiphrenic esophageal diverticulum. JSLS 2008; 12: 104-8.

10. Rosati R, Fumagalli U, Bona S, et al. Diverticulectomy, myotomy, and fundoplication through laparoscopy: a new option to treat epiphrenic esophageal diverticula? Ann Surg 1998; 227: 174-8.

11. Renz EM, Parker MV, Hetz SP. Laparoscopic repair of a large symptomatic epiphrenic esophageal diverticulum. Curr Surg 2002; 59: 190-3.

12. Soares R, Herbella FA, Prachand VN, et al. Epiphrenic diverticulum of the esophagus. From pathophysiology to treatment. J Gastrointest Surg 2010; 14: 2009-15.

13. Baue AE. Functional (motor) disorders of the esophagus. In: International practice in cardiothoracic surgery. Yingkai W, Peters R (eds.). Science Press Dordrecht, Boston, Nijhoff, Beijing China 1986; 478-90.

14. Tamura Y, Funaki Y, Adachi K, et al. A patient with vigorous achalasia and rapid enlargement of an epiphrenic esophageal diverticulum. Intern Med 2015; 54: 1609-12.

15. Sadasivan CS, Umapathy A. Epiphrenic diverticulum of the oesophagus complicated by the impaction of a foreign body. Thorax 1962; 17: 267-70.

16. Jang KM, Lee KS, Lee SJ, et al. The spectrum of benign esophageal lesions: imaging findings. Korean J Radiol 2002; 3: 199-210.

17. Herbella FM, Patti MG. Achalasia and epiphrenic diverticulum. World J Surg 2015; 39: 1620-4.

18. Granderath FA, Pointner R. Laparoscopic transhiatal resection of giant epiphrenic esophageal diverticulum. Dis Esophagus 2007; 20: 353-7.

19. Palanivelu C, Vij A, Rajapandian S, et al. Laproendoscopic single site oesophageal diverticulectomy. J Minim Access Surg 2013; 9: 128-31.

20. Rice TW, Goldblum JR, Yearsley MM, et al. Myenteric plexus abnormalities associated with epiphrenic diverticula. Eur J Cardiothorac Surg 2009; 35: 22-7.

Received: 30.09 .2015 , accepted: 17.11.2015 